http://dx.doi.org/10.18359/ravi.1822

\title{
Modelo Gavilán para el desarrollo de competencias en el manejo de información a través de Google Drive. Una experiencia innovadora ${ }^{1}$
}

Marbelys Elizabeth Cánchica de Medina²

Universidad Nacional Experimental "Francisco De Miranda" (UNEFM), Venezuela

Recibido, abril 25 de 2016

Concepto evaluación, junio 08 de 2016

Aceptado, junio 30 de 2016
Referencia: Cánchica de Medina, M. (2016). "Modelo Gavilán para el desarrollo de competencias en el manejo de información a través de Google drive. Una experiencia Innovadora”. Revista Academia y Virtualidad, 9, (2), 10-26

\section{Resumen}

El presente documento tiene como objetivo presentar una sistematización de experiencias en la asignatura "Introducción a las TIC" del Posgrado en Gerencia Pública en la Universidad Nacional Experimental Francisco de Miranda (UNEFM). Las categorías de análisis corresponden a Google Drive mediante el Modelo Gavilán, en el cual se evidenció productividad e integración de los participantes del curso en términos de trabajo colaborativo. Esto contribuyó a la construcción compartida del proceso y del producto de las investigaciones desarrolladas sobre temas específicos del área en formación. Los resultados muestran el aprovechamiento de la potencialidad comunicativa e interactiva de Google Drive, al contribuir al desarrollo de actividades síncronas y asíncronas entre los integrantes de cada equipo, mediante un conjunto de pasos y sub-pasos que orientaron el alcance de competencias en el manejo de la información (CMI) para la construcción de conocimientos emergentes a partir de procesos investigativos en la web.

Palabras clave: computación en la nube, Modelo Gavilán, Google Drive, gerencia pública, competencias en el manejo de información, trabajo colaborativo.

\footnotetext{
${ }^{1}$ Artículo de investigación, aprobación institucional (UNEFM).

${ }^{2}$ Doctora en Innovaciones Educativas; Docente Universidad Nacional Experimental "Francisco de Miranda"; Adscrita al Departamento de Tecnología Educativa, Falcón, Venezuela 4101. marbelysc@gmail.com
} 


\title{
Gavilan Model to develop skills in information management by Google Drive. An innovative experience
}

\begin{abstract}
This paper offers a systematization of experiences during the 'Introduction to ICTs' of Public Management Postgraduate at the Universidad Nacional Experimental Francisco de MirandaUNEFM. Analysis categories correspond to Google Drive shared by Gavilan Model, where productivity and integration were supported by players in terms of collaborative work. This helped to share processes and products of researches on specific topics of training area. The results show the Google Drive as a useful tool with communicative and interactive prospective, providing development of synchronous and asynchronous activities among the members of each team by a set of steps and sub-steps that guided the scope of skills in information management (SIMs) in order to develop emerging knowledge from research processes in the web.
\end{abstract}

Keywords: cloud computing, Gavilan Model, Google Drive, public management, skills in information management, collaborative work.

\section{Modelo Gavilán para o desenvolvimento de competências na manipulação de informação através de Google Drive. Uma experiência inovadora}

\section{Resumo}

O objetivo do presente artigo tem o intuito de apresentar uma sistematização de experiências no curso "introdução às TIC" do Pós-grau em Gerência Pública na Universidad Nacional Experimental Francisco de Miranda (UNEFM). As categorias de análise correspondem a Google Drive mediante o Modelo Gavião, no qual evidenciou-se produtividade e integração dos participantes do curso no que tem a ver com trabalho colaborativo. O que contribuiu à construção compartida do processo e do produto das pesquisas desenvolvidas sobre temas específicos da área em formação. Os resultados mostram o aproveitamento da potencialidade comunicativa e interativa de Google Drive, ao contribuir no desenvolvimento de atividades síncronas e assíncronas entre os integrantes de cada equipe, por meio de um conjunto de passos e sob passos que guiaram o alcance de competências na manipulação da informação (CMI) para a construção de conhecimentos emergentes a partir de processos de pesquisa na web.

Palavras chave: computação na nuvem, Modelo Gavilán, Google Drive, gerência pública, competência na manipulação de informação, trabalho colaborativo. 


\section{Introducción}

Resulta evidente la creciente importancia que se le ha otorgado a las tecnologías de la información y comunicación (TIC) en la sociedad actual. En el plano educativo, diferentes organismos internacionales se han ido sumando para promover su integración en los entornos académicos; tal es el caso de la Oficina Regional de Educación para América Latina y el Caribe en cooperación con la Organización de las Naciones Unidas para la Educación, la Ciencia y la Cultura (OREALC/ UNESCO), el Programa de Naciones Unidas para el desarrollo (PUND), la Organización de los Estados Ibero-Americanos para la Ciencia y la Cultura (OEI), entre otros.

El PNUD (2012) reconoce que las TIC son herramientas que facilitan el proceso de democratización y articulación de redes de conocimiento, dado que permiten difundir de manera masiva y sistemática el conocimiento de la ciencia, la tecnología e innovación, estimulando el pensamiento científico y el interés de la sociedad hacia estos temas. La OEI (2010), en el documento "La educación que queremos para la generación de los bicentenarios", plantea 28 metas que deben ser cubiertas para 2021, destacando la necesidad de orientarse hacia la calidad de la educación y el currículo escolar, así como el fortalecimiento profesión docente. Entre los niveles de logro se estima que "[...] en el 2021, los profesores y los alumnos utilizan el computador de forma habitual en el proceso de enseñanza y aprendizaje" (p.153).

El acceso a una educación de calidad se constituye, asimismo para la UNESCO (2014), en un derecho fundamental de todas las personas que se enfrentan a la dimensión del cambio paradigmático del siglo XXI, por lo cual demanda al sistema educativo: la actualización de prácticas y contenidos acordes a la nueva sociedad de la información, la incorporación de las TIC al currículum escolar, así como la adecuación de la formación inicial y en servicio de los docentes. La renovación de tales estrategias las justifica al considerar que las recientes estadísticas muestran que en América Latina y el Caribe en los últimos 20 años, las TIC siguen ocupando un lugar marginal en las aulas.

Es importante asimismo destacar que las políticas venezolanas también han reconocido la relevancia de las TIC, por lo cual, entre los objetivos del Plan de la Nación (Plan Patria, 2013), prevé “[...] garantizar la democratización y apropiación del conocimiento del pueblo en materia de equipos electrónicos y aplicaciones informáticas en tecnologías libres a través de programas educativos en los centros universitarios, técnicos, medios y ocupacionales" (p. 11).

En este orden de ideas, se vislumbra que representa una acertada opción el incluir contenidos vinculados tecnologías de la información y comunicación en procesos de formación en todos los niveles educativos. Desde esta perspectiva, el posgrado de Gerencia Pública de Universidad Nacional Experimental "Francisco de Miranda", ha establecido la necesidad de ofrecer un curso de nivelación que busca proveer al participante las herramientas básicas que le permitan desenvolverse con mayor eficacia en su escolaridad. Entre las asignaturas que componen este primer periodo académico se encuentra "Introducción a las Tecnologías de la Información y Comunicación" con el propósito de iniciar a los participantes en los conceptos fundamentales de la computación y a sus aplicaciones más comunes, de tal manera que les permita utilizar el computador personal como herramienta en el desarrollo de sus actividades.

El uso de las TIC sin dudas ocupa un papel preponderante como eje dentro de las herramientas que propone la corriente de la Nueva Gestión Pública (NGP), no sólo para permitir o facilitar los objetivos de mayor eficiencia y eficacia en los quehaceres del sector público, sino también como herramienta para fomentar la transparencia y la rendición de cuentas mediante la participación 
ciudadana. Los procesos en la administración pública indiscutiblemente se están viendo modificados, tanto en el interior de los organismos del Estado como en términos de la relación con los ciudadanos, ante la presencia de nuevas funciones y servicios en línea. Esta noción es de particular interés para la realidad venezolana, teniendo en cuenta las estrategias que se plantean en la Ley Infogobierno (2013). En el marco de estas ideas, es relevante que gerentes y sus recursos humanos apliquen competencias para

"[...] la abstracción, el análisis y la síntesis; la organización y planificación de su tiempo; comunicarse de forma oral y escrita; habilidad para el uso de tecnologías de la información y de la comunicación; habilidades para buscar, procesar y analizar información procedente de fuentes diversas; capacidad para identificar, plantear y resolver problemas; capacidad de aplicar los conocimientos en la práctica; capacidad para tomardecisiones; competencias interpersonales; capacidad para trabajar en equipo; compromiso ético; capacidad crítica y autocrítica; capacidad de investigación; capacidad creativa (Bonina, 2005).

Desde ese punto de vista, las instituciones educativas deben procurar el desarrollo de habilidades no sólo operacionales e informacionales formales correspondientes al manejo, manipulación y uso de hardware y software, sino también habilidades de tipo informacionales sustanciales referentes a la búsqueda, selección, procesamiento y evaluación de información y habilidades estratégicas relativas al uso de fuentes y medios para el logro de objetivos en pro del bienestar social (Gutiérrez, Ariza y Jaramillo, 2014).

Ahora bien, en el marco de la Ley Infogobierno (2013), conviene considerar otro criterio, y es precisamente el que se plantea en su artículo 34, "En las actuaciones que se realicen con el uso de las tecnologías de información, sólo empleará programas informáticos en software libre y estándares abiertos..."
De acuerdo a los planteamientos expuestos, se identificó que el diseño instruccional de la asignatura "Introducción a las TIC" estaba descontextualizado de la realidad y sus exigencias, dado que: a) planteaba el estudio de herramientas ofimáticas privativas; b) el uso de internet con un sentido instrumental; c) actividades didácticas no significativas para el aprendizaje situado. Por otra parte, aunque se pudo detectar que los alumnos del curso, en su mayoría, han nacido rodeados de tecnología y la incorporaran a cada una de las actividades diarias, tales como consultar el saldo bancario por internet, solicitar una cita electrónica con el banco, gestionar cupos en divisas por el sistema del estado, pagar electrónicamente servicios públicos, existe una gran variedad de herramientas de fácil acceso, con valiosos beneficios a todos los campos del saber que no son masivamente utilizadas por éstos, tal como Google Drive.

Google Drive permite redefinir el manejo de aplicaciones ofimáticas en línea, sustentado en el concepto computacional de la nube. Con esto se propicia cambios en los modos de crear, almacenar, modificar, compartir y acceder a los archivos que se generen, planteando actividades de trabajo colaborativo a partir de la interacción síncrona y asíncrona de diversos usuarios sobre un mismo archivo.

En el caso de la unidad II, se planificó el abordaje de la ofimática en línea a través de la herramienta, y para las búsquedas en Internet, se consideró necesario trabajar transversalmente con los estándares de competencias de alfabetización informacional, en este caso con la estrategia del Modelo Gavilán (González y Sánchez, 2007). De allí la motivación de presentar la sistematización de la experiencia realizada, sus resultados y las lecciones aprendidas en aras de declarar nuestra contribución al conocimiento. 


\section{El Modelo Gavilán y el concepto de computación en la nube}

La experiencia con estudiantes regulares tanto de pregrado con posgrado, ha evidenciado que la realización de sus investigaciones suele recoger datos de internet, que pocas veces suele aplicar competencias adecuadas para el manejo de información (CMI), habilidades indispensables del siglo XXI. En este sentido, se determinó la necesidad de hacer uso instrumental de un motor de búsqueda, tal como lo es Google, pero en combinación con un modelo que orientara la resolución efectiva de problemas de información a partir de una indagación sistematizada.

El Modelo Gavilán se compone de cuatro (4) pasos principales, el cual se utiliza para las investigaciones de tipo académico y para la resolución de problemas. Los pasos utilizados tienen sub-pasos, los cuales desarrollan diferentes funciones, todo para lograr la secuencia que se plantea (véase tabla siguiente).
Los autores del modelo han establecido que el objetivo de su aplicación es que los estudiantes desarrollen la CMI, para lo cual sugieren trabajar con profundidad cada uno de los pasos y sub-pasos que lo conforman de manera separada y continua. Han señalado además que el Modelo Gavilán se generó especialmente para el desarrollo adecuado de investigaciones cuya fuente de información principal es internet; sin embargo, puede adaptarse a otras condiciones si así se requiere. Por otra parte, aun cuando el modelo está dirigido a los primeros niveles educativos, los autores de esta experiencia han previsto su utilidad para implementar como actividad didáctica con estudiantes de posgrado.

Tabla 1. Pasos y sub-pasos del Modelo Gavilán

\begin{tabular}{|c|c|c|}
\hline \multicolumn{3}{|r|}{ Modelo Gavilán } \\
\hline & Pasos & Sub-pasos \\
\hline \multirow[t]{5}{*}{1} & \multirow{5}{*}{$\begin{array}{l}\text { Definir el problema de } \\
\text { información y qué se } \\
\text { necesita indagar para } \\
\text { resolverlo }\end{array}$} & $\begin{array}{l}\text { A) definir el problema de información y plantear la pregunta inicial que pueda } \\
\text { ayudar a resolverlo. }\end{array}$ \\
\hline & & $\begin{array}{l}\text { B) identificar, explorar y relacionar los conceptos y aspectos del tema } \\
\text { necesarios para responder la pregunta inicial. }\end{array}$ \\
\hline & & $\begin{array}{l}\text { C) construir el diagrama de un plan de investigación que ayude a seleccionar y } \\
\text { categorizar los conceptos y aspectos del tema más importantes para resolver la } \\
\text { pregunta inicial }\end{array}$ \\
\hline & & $\begin{array}{l}\text { D) formular preguntas secundarias derivadas de la pregunta inicial y del plan } \\
\text { de investigación }\end{array}$ \\
\hline & & E) evaluación. \\
\hline 2 & $\begin{array}{l}\text { Buscar y evaluar } \\
\text { fuentes de información }\end{array}$ & $\begin{array}{l}\text { A) identificar y seleccionar las fuentes de información más adecuadas. } \\
\text { B) aceeder a las fuentes seleccionadas y a la información que contienen. } \\
\text { C) evaluar fuentes encontradas y la información que no contienen } \\
\text { D) evaluación }\end{array}$ \\
\hline 3 & Analizar la información & $\begin{array}{l}\text { A) elegir la información más adecuada para resolver las preguntas secundarias } \\
\text { B) leer, entender, comparar y evaluar la información seleccionada } \\
\text { C) responder las preguntas secundarias } \\
\text { D) evaluación }\end{array}$ \\
\hline \multirow[t]{4}{*}{4} & \multirow{4}{*}{$\begin{array}{l}\text { Sintetizar y utilizar la } \\
\text { información }\end{array}$} & A) recopilar las respuestas a las preguntas secundarias \\
\hline & & $\begin{array}{l}\text { B) elaborar un producto completo que exija aplicar y utilizar los resultados de } \\
\text { la investigación. }\end{array}$ \\
\hline & & C) comunicar los resultados de la investigación a otros \\
\hline & & D) evaluación de cada paso y del proceso \\
\hline
\end{tabular}

Fuente: González y Sánchez (2007) 


\section{Computación en la nube}

A finales del siglo $\mathrm{XX}$ se estiman los primeros antecedentes del concepto "Computación en la nube". Este término se atribuye a John McCarthy quien lo utilizaba para representar a internet (Tecayehuatl, 2013). Su significado se asocia a diversas aplicaciones y servicios que se encuentran almacenados en un lugar no físico, sino indefinido o virtual, además de estar disponibles espaciotemporalmente para sus usuarios.

Con la computación en la nube se crean archivos que se guardan en el sitio, siempre y cuando haya conexión a internet. Estos archivos podrán editarse cada vez que se requiera desde cualquier máquina o dispositivo. Google Drive se basa en el concepto de computación en la nube, por lo cual rompe con la idea tradicional de instalar programas, o de guardar los archivos en diferentes tipos de unidades de almacenamiento, pues será posible acceder a ellos en la medida que haya conectividad a internet.

\section{Metodología}

La sistematización de experiencias es una metodología que permite la interpretación crítica de una o varias experiencias, a partir de su ordenamiento y reconstrucción, permitiendo descubrir, explicitar la lógica del proceso vivido $\mathrm{y}$, construir un conocimiento emergente (Jara, 2012). En este sentido, se pretendió estudiar inicialmente las vivencias de cien (100) estudiantes y cuatro (4) docentes, durante el periodo II-2014, en el contexto del dictado de la unidad II, la asignatura "Introducción a las TIC" del Posgrado Gerencia Pública de la UNEFM, institución que se encuentra ubicada en Santa Ana de Coro, estado Falcón-Venezuela. No obstante, sólo fue posible realizar la indagación con base en una muestra determinada por los estudiantes que permanecieron activos en el lapso previsto.

Las etapas que se siguieron para este proceso estuvieron enmarcadas en la propuesta de Jara (2012), la cual incluye cinco fases, tal como se ilustra en la figura que se presenta a continuación, donde además se explicitan elementos conceptuales del modelo:

1. Punto de partida

2. Preguntas iniciales

3. Recuperación del proceso vivido

4. Reflexión de fondo

5. Puntos de llegada

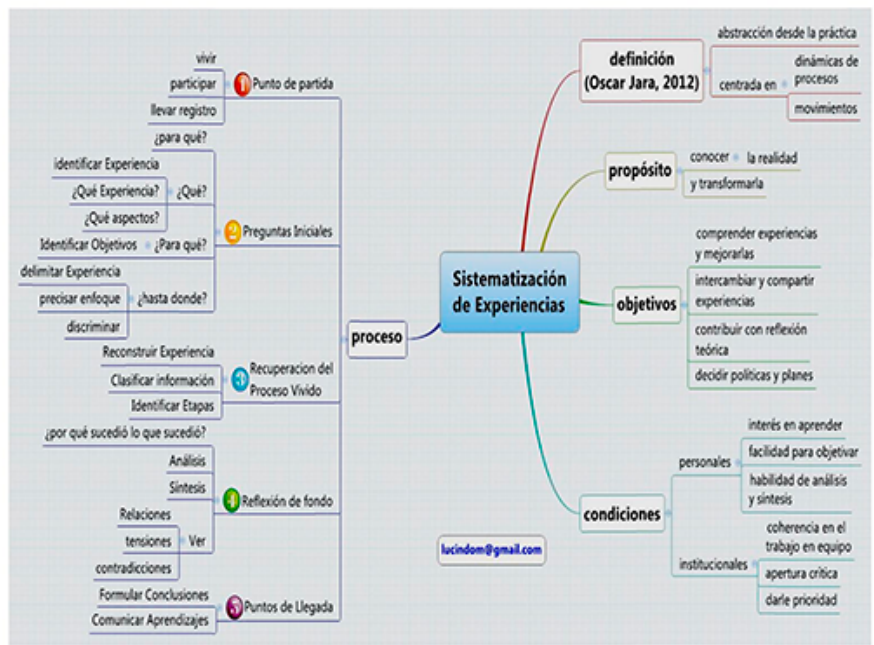

Figura 1. Sistematización de experiencias.

Fuente: Diseños instruccionales para entornos virtuales-CIU-DEDALO. 
En consecuencia, fue necesario rescatar el proceso donde habían estado involucrados los docentes y los estudiantes activos, a fin de evidenciar cómo se había actuado, analizar los efectos de estas intervenciones y el carácter de las relaciones que se habían generado, sin que por ello dejara de considerarse el éxito o fracaso del proyecto en términos de resultados.

\section{Sistematización de la experiencia}

La sistematización realizada sobre las experiencias se ha ceñido al esquema de Jara [9], por ello resulta importante comprender el proceso seguido, el cual se estructuró según las fases señaladas a continuación:

1. El punto de partida: la vivencia propia de la experiencia se constituye en requisito para la aplicación de un proceso de sistematización. Por tanto, es importante destacar que los autores de este documento son la totalidad de los docentes que participaron efectivamente en el diseño e implementación de la materia "Introducción a las TIC" de la Maestría Gerencia Pública de la UNEFM, durante el período II-2014. Por otra parte, es importante señalar que el estudio inició con cien (100) estudiantes distribuidos entre los cuatro (4) docentes.

2. Las preguntas iniciales: a partir del proceso de sistematización se resumen así:

- ¿Para qué queremos sistematizar?

- ¿Qué experiencia queremos sistematizar?

- ¿Qué aspectos centrales de esa experiencia nos interesa sistematizar?

En atención a estas interrogantes se ha orientado la aplicación de la metodología mencionada que apunta hacia lo siguiente. El objetivo de la presente sistematización es que los resultados puedan convertirse en un referente vivencial para fortalecer el proceso del dictado de la asignatura en posteriores oportunidades, en aras de:

a) Mantener la vigencia del diseño instruccional a partir del estudio de herramientas tecnológicas básicas que vayan evolucionando y apareciendo en el tiempo.

b) Contribuir significativamente con el perfil de profesionalización a partir de las actividades didácticas que se planifiquen

c) Aportar conocimientos surgidos precisamente de la reflexión de la experiencia.

d) Compartir aprendizajes.

El hilo conductor entonces se orienta en mitigar las debilidades que presentan los estudiantes de maestría en la elaboración de investigaciones en grupo, al aprovechar las ventajas que ofrece el concepto de computación en la nube y del Modelo Gavilán. Es importante destacar asimismo que de todo el proceso de formación de los maestrantes, en el marco la asignatura "Introducción a las TIC" del Posgrado en Gerencia Pública de la UNEFM, solo se estudia la experiencia que se vincula al desarrollo a la unidad temática II en cada una de sus fases instruccionales.

La información fue recolectada a partir de los registros que genera el aula virtual de la asignatura (foros, tareas y herramienta analítica de la plataforma Moodle), correspondientes a la unidad II. También se consideraron los documentos desarrollados en Google Drive, así como mensajes recibidos en los correos electrónicos de los docentes. En cuanto a los instrumentos utilizados, se pueden mencionar el registro semanal de los docentes, el registro de actividades cumplidas y la ficha de recuperación de aprendizajes. El plan operativo de la sistematización permitió definir tareas por realizar, responsables, procedimientos seguidos $\mathrm{y}$, su cronograma.

3. Recuperación del proceso vivido: con el propósito de reconstruir de forma ordenada lo acontecido, se ha clasificado la información recolectada así como también un conjunto de pantallazos que pretenden sintetizar los registros secuenciales más importantes de la experiencia. Es importante indicar que el proceso instruccional obedeció principalmente a una planificación didáctica preliminar, la cual se ajustó en atención a las tendencias 
tecnológicas actuales y el perfil de egreso. La modalidad de estudio seleccionada para el dictado de la asignatura fue semipresencial (b-Learning), razón por la cual se diseñó y desarrolló un aula virtual bajo la plataforma Moodle para dar paso al proceso de formación correspondiente. En esta oportunidad, en una estructura global sólo se muestran los acontecimientos más significativos en cada etapa de la experiencia.

\section{Plan didáctico}

\section{Objetivo general de aprendizaje}

- Utilizar correcta y pertinentemente las herramientas tecnológicas al servicio de la producción de información y conocimiento vinculada con su área de dominio profesional y de investigación.

\section{Objetivos específicos}

- Aplicar distintos procedimientos de búsqueda, tales como avanzada, especializada $\mathrm{y}$ académica, junto con las alertas en actividades de investigación académica.

- Crear documentos en línea, producto de la sistematización de búsquedas con base en el modelo Gavilán.

\section{Contenidos}

- Tema 1. Búsqueda de información en internet: subtemas: búsqueda avanzada; búsqueda especializada; búsqueda académica; alertas.

- Tema 2. Concepto computacional la nube: edición y publicación de información: subtemas: ofimática en línea; Google Drive; subida de archivos; edición de documentos; edición de hoja de cálculo; edición de presentaciones; cuestionarios; compartir documentos

- Tema 3: Sistematización de búsquedas: subtemas: pasos y sub-pasos del Modelo Gavilán

Actividad de aprendizaje: investigación basada en búsqueda de información en la web con el Modelo Gavilán usando ofimática en línea.

1. Antes de comenzar la actividad, los participantes deben

- Formar grupos de 3 o 4 personas.

- Seleccionar un coordinador.

2. El coordinador del grupo de tres o cuatro integrantes debe:

- Descargar la plantilla "Modelo Gavilán" publicada en el apartado correspondiente a la unidad II, del aula virtual de la asignatura. Recuerda acceder con su clave de usuario.

- Guardar la plantilla indicada en su laptop, PC u otro dispositivo.

- Cargar la plantilla en su cuenta en Google Drive

- Abrir la plantilla con el editor de Google Drive para documentos

- Invitar desde Google Drive a los tres integrantes del grupo y al docente

- Utilizar todas las herramientas que proporciona Google Drive para interactuar con el equipo de trabajo (por ejemplo sesiones de chat).

3. Para alcanzar los objetivos planteados, el participante debe tener en cuenta los siguientes pasos:

- En su correo Gmail, aceptar la invitación del documento compartido por el coordinador del grupo.

- Acceder a la plantilla del Modelo Gavilán, compartida por el coordinador del grupo mediante la invitación recibida en su correo Gmail (opción Abrir documentos).

- Organizarse de manera virtual para la distribución de roles y tareas, a través de las herramientas de Google Drive (correo, chat). Por ejemplo, en la primera fase de la plantilla, es recomendable realizar una lluvia de ideas para llegar a acuerdos con relación al tema de indagación y la información que será desplegada.

- Editar de forma colaborativa el documento recibido, utilizando la herramienta de Google Drive. Cada uno de los integrantes debe identificarse en cada fase donde corresponda realizar su contribución a la tarea del grupo. Recordemos que todo lo que edite el usuario, 
será guardado automáticamente y visualizado por el resto de sus compañeros de grupo en cada una de las siguientes acciones:

- Definir el problema de información (seguir plantilla fase paso 1)

- Buscar y evaluar la información (seguir plantilla fase paso $2 \mathrm{a}$ y $2 \mathrm{~b}$ ).

- Analizar la información (seguir plantilla fase paso 3)

- Sintetizar la información y utilizarla. (seguir plantilla fase paso 4)

4. Luego de culminar con el vaciado de la plantilla, publicar los resultados en el foro II del aula virtual creando un nuevo tema por grupo.
- Cada grupo creará y participará en un foro de discusión sobre el tema investigado.

- Cada grupo hará el cierre al tema creado para su investigación en el mismo foro.

- Cada participante debe dar retroalimentación a los resultados obtenidos de otros dos (2) grupos.

\section{Criterios de evaluación}

Veamos los siguientes elementos que configuran las pautas de evaluación de este ejercicio en la tabla 2.

Tabla 2. Rúbrica de evaluación de la unidad II

\begin{tabular}{|c|c|}
\hline Aspectos por evaluar & Valor \\
\hline $\begin{array}{l}\text { Uso instrumental de ofimática } \\
\text {-Uso de G-Drive } \\
\text { (cargar, invitar, editar, uso de herramientas) }\end{array}$ & \multirow[t]{3}{*}{8 puntos } \\
\hline Construcción del informe (usando Google Drive) & \\
\hline Presentación del resumen (usando Google Drive) & \\
\hline $\begin{array}{l}\text { Búsqueda sistematizada en la web } \\
\text { 'Definición del problema de investigación }\end{array}$ & \multirow[t]{4}{*}{4 puntos } \\
\hline Búsqueda y procesamiento de la información & \\
\hline Abstracción y análisis & \\
\hline Sintesis y presentación de resultados & \\
\hline $\begin{array}{l}\text { Aprendizajes dialógicos } \\
\text { Publicar en el foro }\end{array}$ & \multirow[t]{3}{*}{4 puntos } \\
\hline $\begin{array}{l}\text { Retroalimentación individual con base en los resultados } \\
\text { obtenidos de } 2 \text { grupos }\end{array}$ & \\
\hline - Cierre grupal del tema creado & \\
\hline $\begin{array}{l}\text { Otros aspectos } \\
\quad \text { Comunicación escrita }\end{array}$ & \multirow[t]{4}{*}{4 puntos } \\
\hline Trabajo en equipo & \\
\hline Capacidad crítica & \\
\hline Creatividad & \\
\hline Total & 20 puntos \\
\hline
\end{tabular}




\section{Diseño y desarrollo del aula virtual}

En una segunda fase, se procedió a generar el aula virtual desde la metodología del Aprendizaje Dialógico Interactivo a partir de la discusión, reflexión y aportes de cada uno de los docentes participantes, para lo cual se desarrollaron varias jornadas de trabajo. Los aspectos generales del aula virtual (véase figura 2), presentan instrucciones para el manejo instrumental del aula virtual y aspectos didácticos de la asignatura. El rincón de comunicación incluye noticias en línea en atención a la necesidad de publicar oportunamente determinados eventos $\mathrm{o}$ situaciones no previstas que deban ser conocidas o recordadas a los participantes; y el rincón de interacción comprende consultas y soluciones (espacio para asesorías virtuales asincrónicas), además del cafetín virtual (espacio para socializar informalmente) y la clase virtual (espacio para encuentros síncronos virtuales).

La unidad II se denominó "Búsqueda y edición de información" y se constituyó en el aula virtual como guía introductoria y otras vinculadas al contenido específico en estudio, tales como: opciones de consulta en Google, búsqueda avanzada en Google, Google académico, trucos para la búsqueda en Google, ofimática en línea, construcción de documentos con Google Drive, el Modelo Gavilán y sus plantillas, así como también las instrucciones de la actividad didáctica.

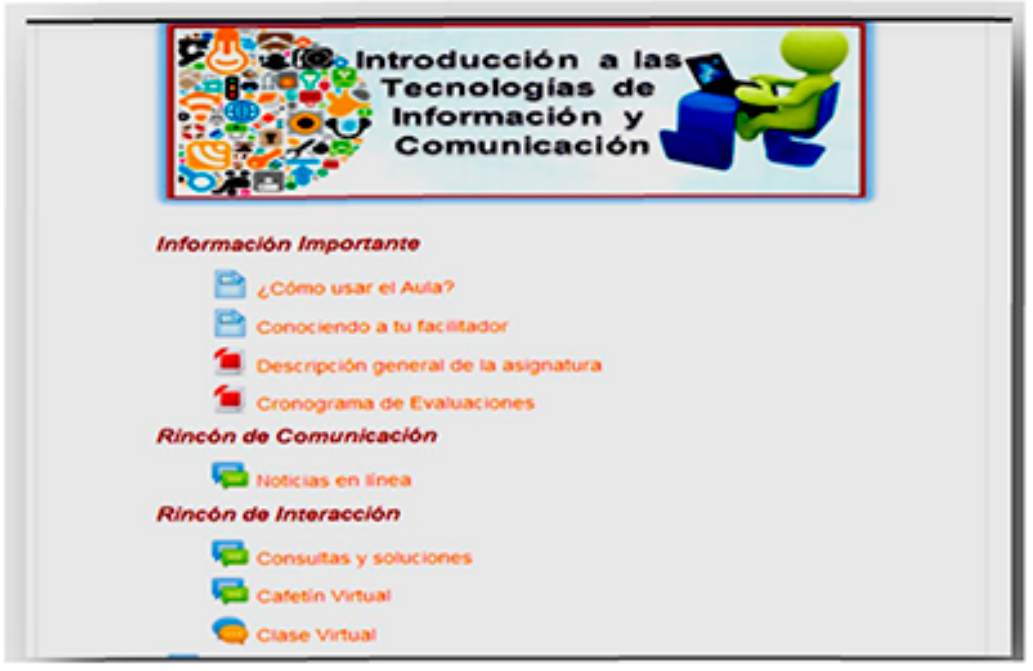

Figura 2. Pantallazo de los elementos preliminares del aula virtual de la asignatura.

\section{Inicio de la experiencia de aprendizaje}

La audiencia del curso de conformó por 100 participantes distribuidos en cuatro grupos con sus respectivos docentes. La audiencia era heterogénea, profesionales que laboran en instituciones públicas del Estado como la Gobernación de Falcón, Hidrofalcón, Corpoelec, Instituciones Universitarias, entre otros. El proceso inicial constó con una fase 0 , desde un encuentro presencial, que se basó en la exploración y uso del aula virtual así como también el abordaje de contenidos introductorios; el resto de las unidades temáticas se desarrollaron virtualmente, incluyendo las unidades II y III.

Con respecto a la Unidad II, y siguiendo las instrucciones establecidas, el coordinador de cada grupo procedió a descargar del aula virtual la plantilla del Modelo Gavilán, la montó en Google Drive y la envió a sus compañeros y docentes para compartirlo virtualmente (véase figura 3). 
Una vez que cada estudiante recibiera la invitación en su correo, iniciaba el tiempo para comenzar el proceso de elaboración colaborativa en línea del documento de investigación, según el tema de preferencia de cada grupo. Por otra parte, también se daba paso a que el docente pudiera entonces emprender el monitoreo del trabajo que estaba realizando cada grupos haciendo clic en el historial de Google Drive (véase figura 4).

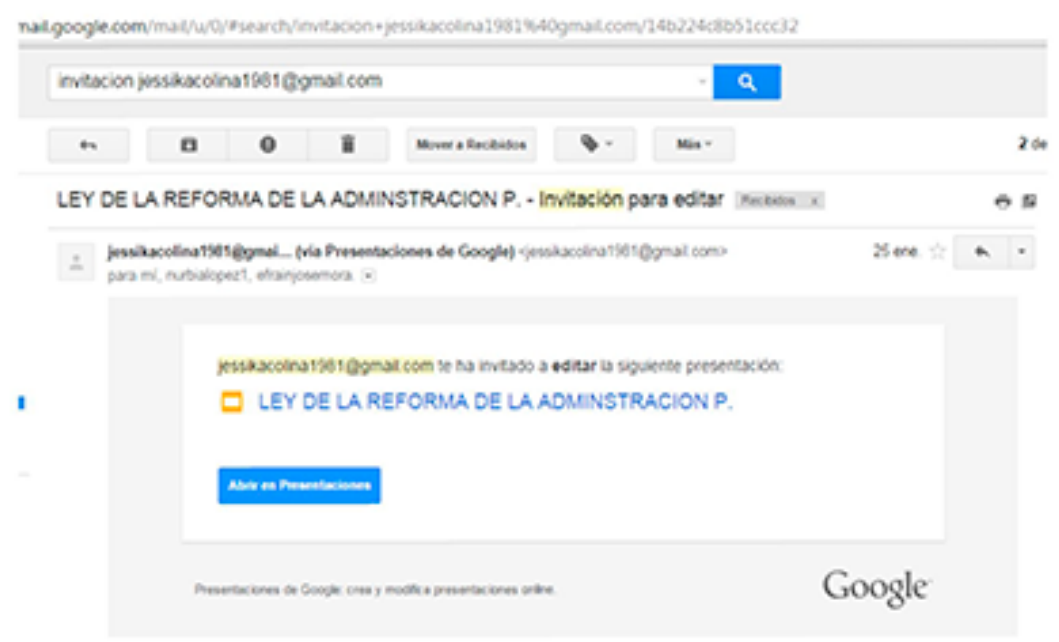

(2)

Figura 3. Pantallazo ejemplo que muestra la invitación para editar con Google Drive.

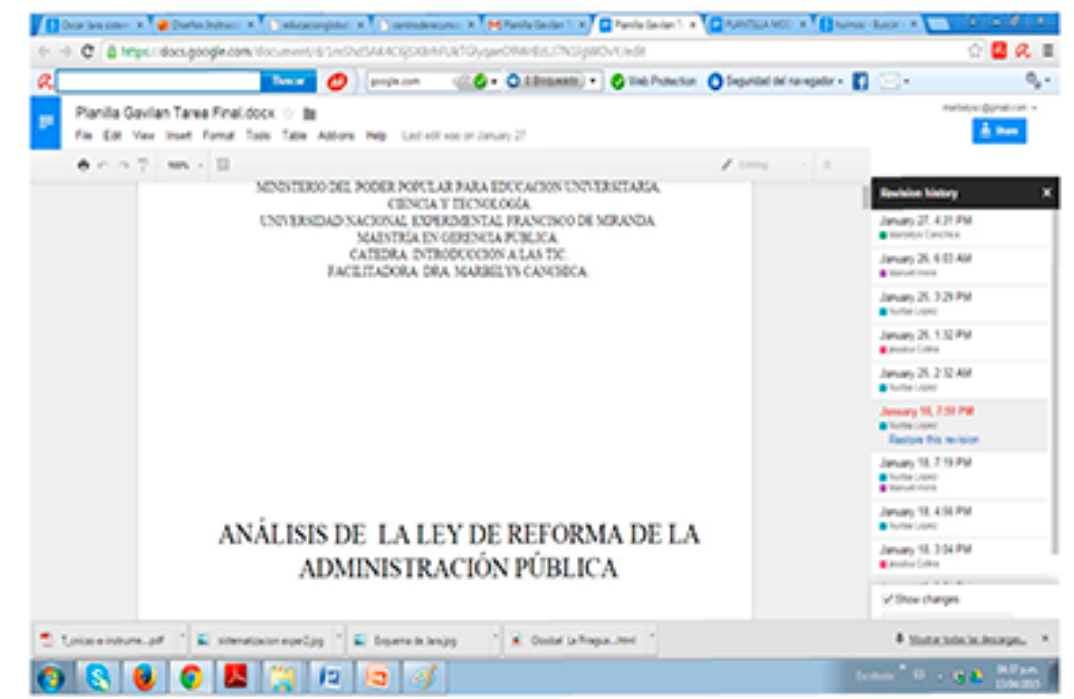

Figura 4. Pantallazo ejemplo que muestra en la ventana izquierda el historial de las contribuciones que realizan los participantes en la edición del documento. 
Durante esta primera fase, ocurrieron tres eventos particulares, que exigieron a los docentes establecer algunas medidas de atención:

a) Coordinadores de grupos que realizaban de manera solitaria todo el trabajo, guardándolo y compartiéndolo en Google Drive.

b) Participantes, que realizaban las tareas del coordinador compartiendo la plantilla con todos, pero trabajando de manera individual c) Presentación de excusas al no realizar las actividades a tiempo por suponer que requería de la reunión física entre los participantes del equipo

d) Montaje en el foro del aula virtual un documento con el trabajo final en formato pdf. Esta situación demandó una relectura y ajuste de las instrucciones escritas, y exigió la atención y asesoría por separado de determinados grupos, los cuales finalmente reiniciaron efectivamente con sus actividades.

\section{Resultados. Productos de la experiencia de aprendizaje}

Una vez culminadas las investigaciones, cada coordinador de cada grupo compartió un enlace contentivo del informe grupal desarrollado y una presentación resumen del mismo.

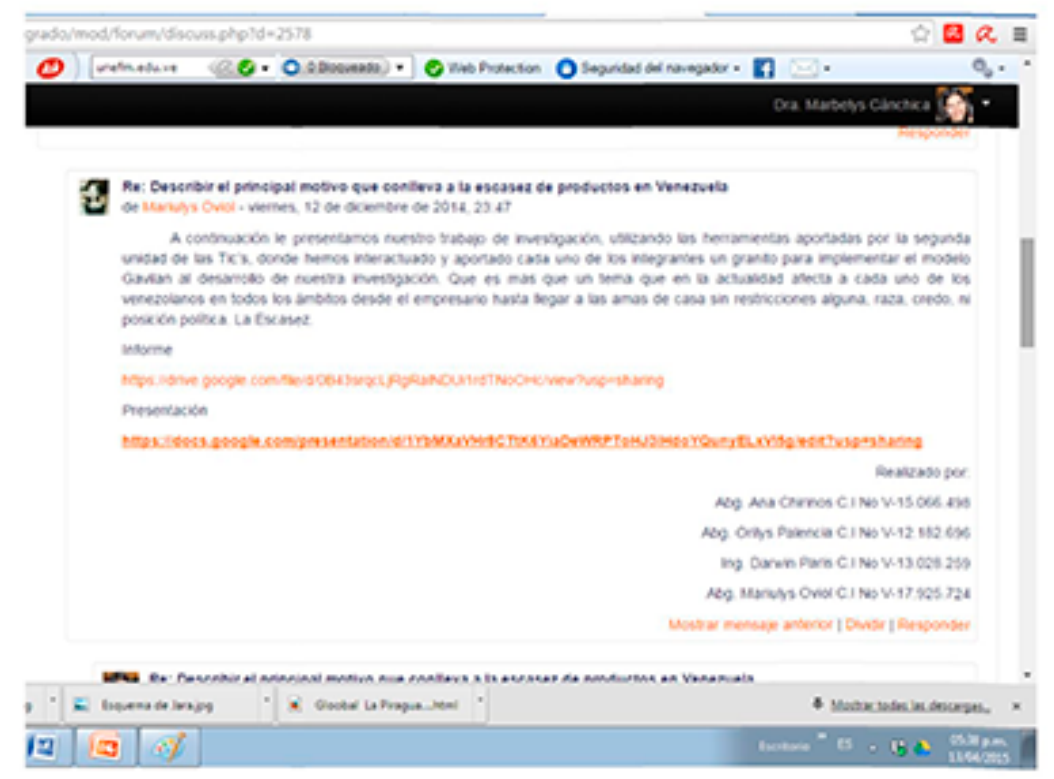

Figura 5. Pantallazo ejemplo que muestra la publicación en la nube de los trabajos realizados. 


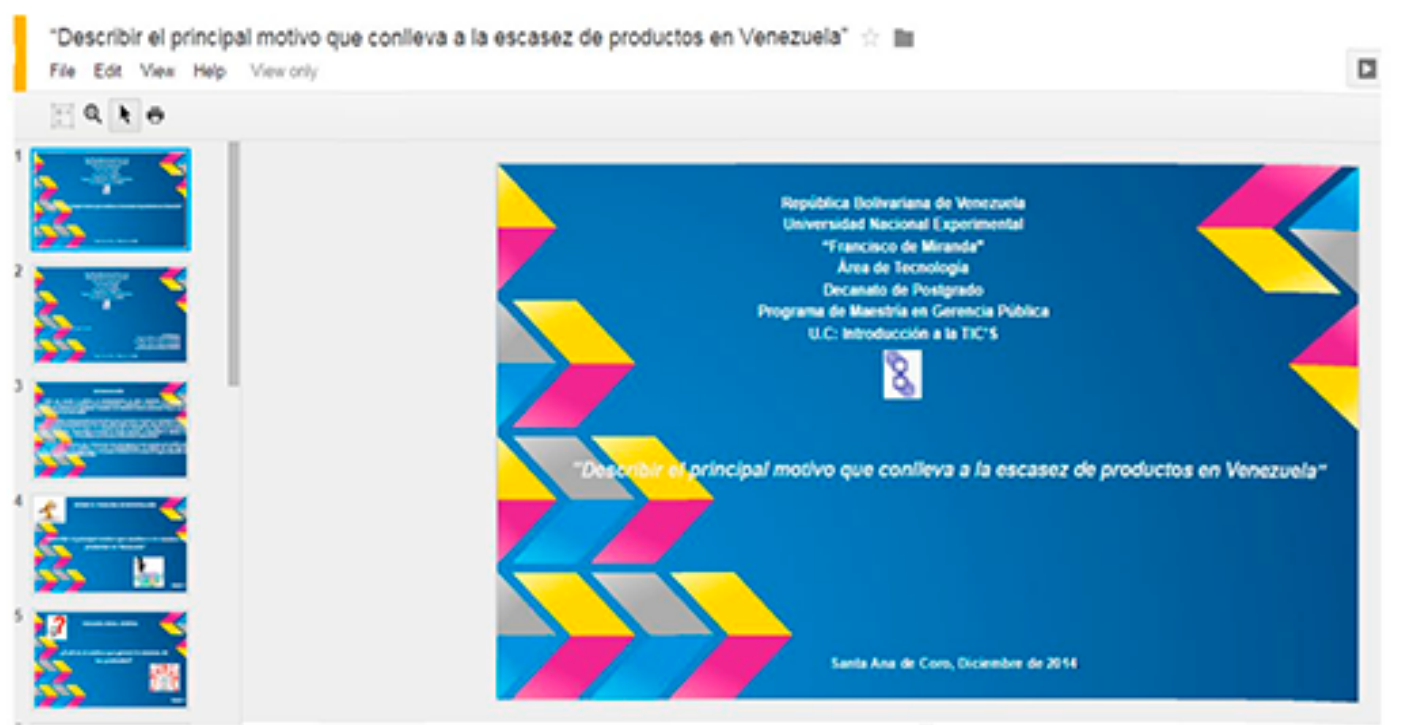

\section{Figura 6. Pantallazo ejemplo de una presentación en la nube.}

Por cada recurso publicado, en los temas del foro del aula virtual, los participantes hicieron sus contribuciones a través de comentarios, valoraciones o críticas.

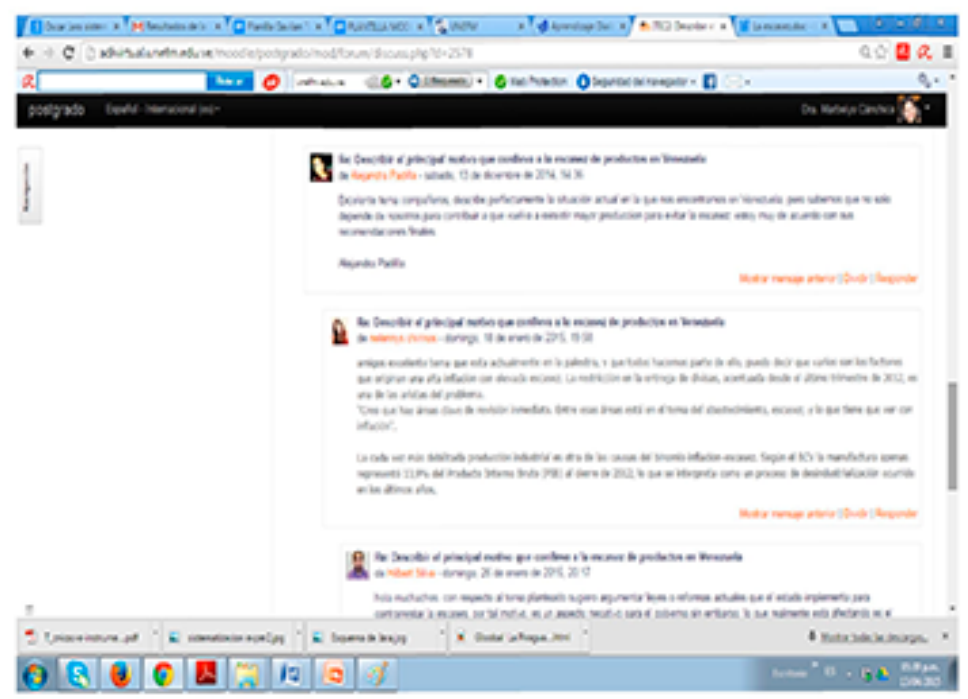

Figura 7. Pantallazo ejemplo de foro temático para la discusión de los trabajos realizados.

Los estudiantes que siguieron efectivamente la investigación a partir del Modelo Gavilán, presentaron los productos correspondientes con cada uno de los pasos y sub-pasos. Para mostrar evidencias de esta declaración, se ha generado una tabla, en la cual se recoge el proceso seguido en uno de los trabajos realizados, donde se puede observar la aplicación de cada una de las fases. 


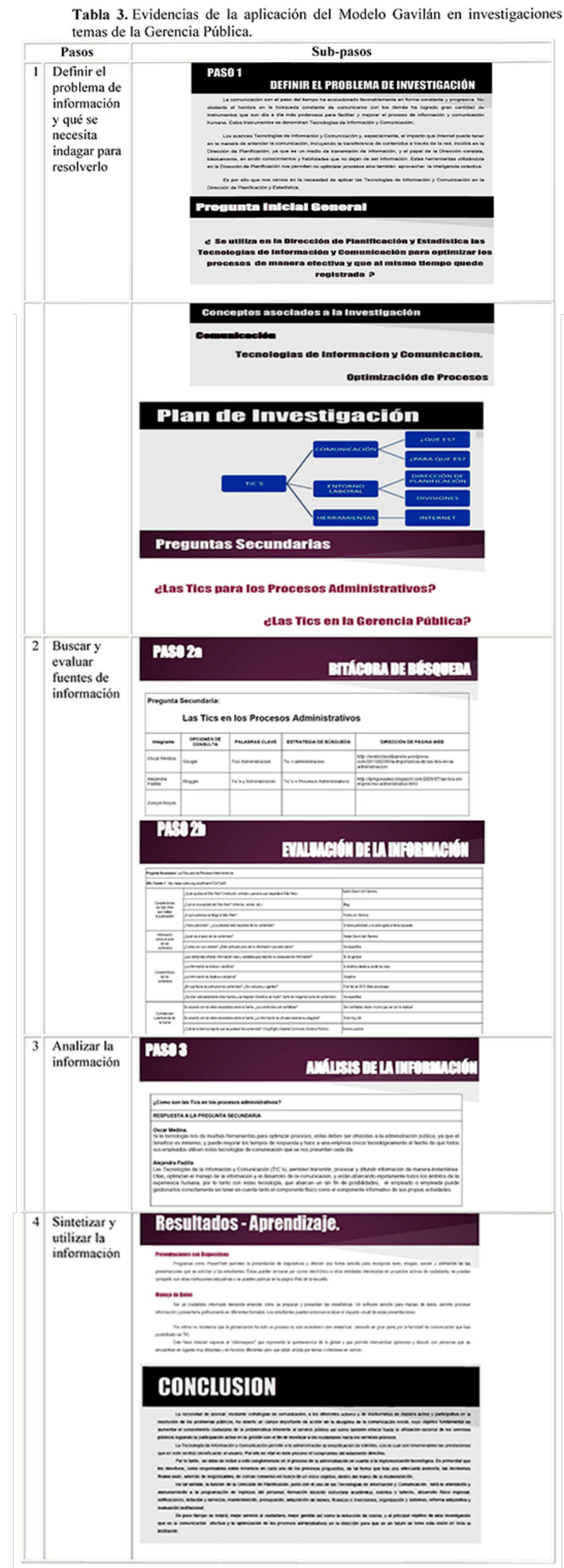




\section{Discusión. Cierre de la experiencia}

Los temas de investigación abordados por los participantes del curso fueron los siguientes:

- Cobro de aranceles para la obtención de pasaportes para niños, niñas y adolescentes, en contra-posición con el artículo 9 de la LOPNNA

- Creación de una sala de lactancia y guardería para los hijos e hijas de las trabajadoras y trabajadores de Mercal C.A. Falcón

- Hipertensión arterial

- Importancia de la administración pública

- Importancia del uso de las técnicas de la información y la comunicación en la gestión eficiente de la administración pública

- La escasez en Venezuela

- Las TIC como herramientas en la gerencia pública

- Motivación como agente influyente en el desempeño laboral del personal administrativo del instituto regional de la mujer, sede Coro

- Análisis de la reforma de la ley de la administración pública en Venezuela

- Sistematización de proyectos sociointegradores en las universidades politécnicas territoriales de Venezuela

- Uso de las TIC en la dirección de planificación en la UNEM

- Uso de las TIC para superar las barreras administrativas a la inversión

- Influencia de las telecomunicaciones en la gerencia pública.

En este sentido, es importante destacar que de los cien (100) estudiantes solo participaron efectivamente sesenta (60), pues diez (10) abandonaron, diez (10) reprobaron y el resto participó en pruebas de suficiencia. Para el cierre de la experiencia, cada participante expresó sus opiniones y valoraciones del proceso vivido en el foro cierre de la unidad II del aula virtual. Al respecto se ha recogido una muestra de los testimonios:

[...] Es mi primera vez con Google Drive, me será muy útil de ahora en adelante, puesto que ahora puedo guardar cosas en lo que llamamos "nube" sin necesidad de usar pendrive, $\mathrm{Cd}$, etc.; y siempre estará disponible con el uso de internet.

[...] El equipo comprendió que utilizar Google Drive es un recurso que nos acorta las distancias, nos abre los espacios, nos permite construir en colectivo y apreciar con ópticas diferentes un tema en común, además nos permite compartir saberes y crear muchos nuevos.

[...] La actividad en Google Drive y el Modelo Gavilán es realmente una experiencia nueva, única e interesante, que nos permite desarrollar herramientas útiles para abordar, discutir y difundir diferentes tópicos sobre la gerencia pública.

[...] Aplicando un PNI se puede decir, en lo positivo: está la interacción en el grupo, el aprendizaje obtenido, el trabajo en equipo de una manera diferente; negativo: el tiempo, el acceso al aula presencial para fortalecer el acompañamiento a quienes presentaron debilidades por la poca experiencia en esta forma de estudio entre otras cosas que se escapan de las manos tanto del instructordocente como del participante-estudiante; interesante: la forma de intercambiar con los grupos el aprendizaje en un lapso muy corto, la flexibilidad para realizar tareas en horarios nocturnos por las limitaciones en relación con las responsabilidades individualidades.

\section{La reflexión de fondo}

El curso de inducción del Posgrado de Gerencia Pública de la UNEFM, denominado "Integración a las TIC", permitió la aplicación del Modelo Gavilán en la nube como herramienta para la elaboración de investigaciones colaborativas. En el desarrollo de la experiencia se identificaron factores que lo potenciaron o lo inhiben. Entre los factores potenciadores se identificaron los siguientes:

a) Conocimientos previos en el área de informática básica por los participantes

b) Actitud positiva ante el uso de las tecnologías 
c) Seguimiento de instrucciones por parte de los participantes sumando aportes de creatividad a sus tareas

d) Organización interna de los equipos para establecer pautas y roles

e) Uso de distintos medios para la interacción docente-alumno

f) Construcción colectiva del conocimiento

g) Participación efectiva de los miembros del grupo en la ejecución del informe.

Con respecto a los factores inhibidores, se pueden señalar los siguientes:

a) Inicialmente los estudiantes discutían sobre la actividad durante los encuentros presenciales

b) Algunos estudiantes no participaban continuamente por carecer de conexión a internet desde sus escenarios

c) Los laboratorios de posgrado de la UNEFM tienen problemas de conexión y no están disponibles para los estudiantes

d) Falta de experiencia en modalidad de aprendizaje semipresencial

d) Estudiantes que no accedían al aula virtual

e) Poco uso del aula virtual para asesorías

f) Participantes que dejaron de realizar la actividad a última hora.

Por consiguiente, se identifica que los atributos mencionados como factores potenciadores o inhibidores del proceso de innovación desarrollado pueden ubicarse en las siguientes dimensiones:
a) Personal
b) Social
c) Institucional
d) Académico y Profesional

\section{Conclusiones. Puntos de llegada}

En líneas generales cabe destacar que la ofimática, desde Google Drive, favorece el seguimiento del proceso de escritura colaborativa en línea. Permite que los miembros involucrados gestionen sus procesos de escritura y que los profesores puedan hacer un seguimiento pormenorizado. Google Drive, como herramienta para la producción de informes y su resumen, permite indagar sobre los productos y procesos previos que los estudiantes ponen en marcha cuando escriben colaborativamente; no obstante, se abre el camino para la investigación sobre la forma como la escritura colaborativa en línea puede aparecer como espacio ameno e innovador entre profesores-estudiantes y entre pares.

Por tanto, existen dimensiones que favorecen o no el uso de Google Drive para la construcción de informes colaborativos desde la base del Modelo Gavilán, a saber: la Personal, Social, Institucional y Académico profesional. Esto significa que la implementación de un proceso similar requiere la atención de condiciones multidimensionales que están directamente entrelazadas, tales como que el docente y estudiante tenga cualidades particulares que favorezcan el uso efectivo de la herramienta, las relaciones sociales entre los pares, la infraestructura con que cuenta la universidad y los participantes, y las metas personales como profesionales de excelencia que puedan establecerse.

La experiencia ha sido significativa para los estudiantes puesto que han participado con actitudes positivas y también han manifestado el deseo de seguirla incorporando en tareas similares, lo cual ha favorecido el alcance de competencias en el manejo de información para la elaboración de investigaciones sistematizadas basadas en búsquedas desde la web y el aprovechamiento de la potencialidad de herramientas a partir del concepto de la nube con fines colaborativos e interactivos. Por tanto, se declara que el contenido es apto para un curso introductorio, pues se reconoció que los estudiantes no tenían experiencia con esta tecnología ni con un modelo que orientara las búsquedas en internet, lo cual es fundamental como herramienta para fortalecer el proceso de indagación que requieren los estudios de otros niveles. 


\section{Referencias}

Bonina, C. (2005).Tecnologías de información $\mathrm{y}$ nueva gestión pública: experiencias de gobierno electrónico en México. Recuperado de: http://www.iapqroo.org.mx/ website/biblioteca/tecnologias $\% 20 \mathrm{de} \% 20$ informacion $\% 20 y \% 20$ nueva $\% 20$ gestion $\% 20$ publica.pdf

González, L. y Sánchez, B. (2007). Guía para utilizar el Modelo Gavilán en el aula. Recuperado de: www.eduteka.org/ modulos/1/1/

Gutiérrez, L.; Ariza, L. y Jaramillo, A. (2014). Estrategias didácticas en el uso y aplicación de herramientas virtuales para el mejoramiento en la enseñanza del cálculo integral. En: Revista Academia y Virtualidad, 7, (2), 64-75.

Jara, O. (2012). Orientaciones teórico-prácticas para la sistematización de experiencias. Disponible en: centroderecursos.alboan.org/ ebooks/0000/0788/6_JAR_ORI.pdf

Ley Infogobierno (2013). Gaceta Oficial República Bolivariana de Venezuela $\mathrm{N}^{\circ}$ 40.274. Recuperado de: www.cnti.gob.ve/images/ stories/documentos_pdf/leydeinfogob.pdf

Mora, L. (2013). Diseños instruccionales para entornos virtuales-CIU-DEDALO. Recuperado de: dedalo-ciu-di-lmora.blogspot. com/p/introduccion.html

OEI (2010). Metas Educativas 2021. La educación que queremos para la generación de los bicentenarios. Disponible en: www.oei. es/metas2021.pdf

PNUD (2002). Informe sobre desarrollo humano en Venezuela. Las tecnologías de la información y comunicación al servicio del desarrollo ISBN: 980-6524-03-9. Impreso en talleres offset.
Tecayehuatl, E. (2013). El origen de: El cómputo en la nube. Recuperado de: www. fayerwayer.com/2012/01/el-origen-de-elcomputo-en-la-nube/.

UNESCO (2014). Informe sobre tendencias sociales y educativas en América Latina. IPP-UNESCO, sede Regional Buenos Aires (2014). 